\title{
Analysis of cellulose acetate membranes for lab-on-a-chip applications
}

\author{
H. Mohamed, ${ }^{*}$ D. H. Szarowski, ${ }^{*}$ M. G. Spencer, ${ }^{* *}$ D. L. Martin, ${ }^{*}$ M. Caggana,, ${ }^{* * *}$ and J. N. \\ Turner ${ }^{* * * *}$
}

*Wadsworth Center, New York State Department of Health, PO Box 509, Albany, NY 12201

**Cornell University, Ithaca, NY 14853

***The University at Albany, Albany, NY 12201-0509

The miniaturization of microfluidics devices and total analysis systems for chemical and biological applications has been motivated by the decrease in sample size and processing time, while improving the sensitivity. However, sensitivity depends in most cases on the purification or enrichment of the sample, which is currently performed prior to loading onto a device. We developed a process to integrate polymer membranes onto silicon wafers using a standard microfabrication process to add filtration capability on chip. The membranes are biocompatible, have good structural integrity and good adhesion to the substrate.

Polymer solutions were prepared by dissolving cellulose acetate (CA) (39.8 wt \% acetyl content, $\mathrm{Mn}=30,000$ ), in $\mathrm{N}, \mathrm{N}$-dimethylacetamide (DMAc) at various concentrations: $10 \%, 25 \%$, and $40 \%$ $(\mathrm{w} / \mathrm{v})$. The polymer solution was spun on the wafer at $2000 \mathrm{rpm}$ for $20 \mathrm{sec}$. After spinning, the coated wafers were immersed in a water bath for $10 \mathrm{~min}$, removed and allowed to air dry. Scanning electron microscopy was used to study the membranes internal structure change with the different polymer solution concentrations.

SEM pictures revealed the membranes structure: two thin skin layers (20-30nm thick) containing the bulk material with voids ranging hundreds of $\mathrm{nm}$ to several microns in size. The membranes ranged in total thickness from 25 to $70 \mu \mathrm{m}$. SEM also revealed a change in the internal structure of the membrane using different polymer solution concentrations (Figure1). All three membranes exhibited skin layers. The internal structure of the $10 \%$ was completely filled with relatively small voids (200$300 \mathrm{~nm}$ ) (Figure 1a). The 25\% membranes had large voids aligned and were a few microns wide and up to tens of microns long spanning across the entire thickness of the membrane without penetrating the skin layer (Figure1b). The 40\% membrane structure had smaller number of voids that spans across a smaller percentage of the membrane's thickness (Figure 1c). To investigate the contribution of these internal voids in the filtration process, we tested membranes made of $25 \% \mathrm{CA}$ in DMAc that had voids (Figure 2a), or were treated chemically by incorporating carboxymethylcellulose in the water bath) and had no voids. The rejection characteristics were similar from both membranes suggesting that these voids do not contribute to the molecular weight cut-off (MWCO). To further investigate the contribution of the voids in filtration, a solution containing a fluorescent molecule, which was smaller than the MWCO of the $25 \%$ membrane (3,6-diaminoacridine hemisulfateproflavine, MW $258 \mathrm{Da}$ ), was added on one side of the membrane and left over night in the dark. The dye distribution in the membrane was imaged by confocal laser scanning microscopy, 25 optical slices spaced at $1 \mu \mathrm{m}$ in the z-dimension, were collected and presented as a maximum intensity value projection using BIO-RAD ${ }^{\mathrm{TM}}$ and ANALYZETM software. We observed fluorescence through the whole membrane except for the areas where there were large voids, suggesting that molecules did not penetrate into the voids (Figure 3). 
These experiments revealed the change in membrane structures with varying polymer concentrations. Additionally, it showed that for one membrane structure (the 25\%), the presence or absence of the voids did not change the MWCO suggesting that the rejection occurs at, or very close to the membrane surface (skin layer).

\section{References}

[1] A. P. Russo et al., Separation Science and Technology. 39 (2004) 2525.

[2] A. P. Russo et al., Bioinformatics, Proteomics, and Functional Genomics Conference Proceedings. (2002) 171.

[3] H. Mohamed et al., Separation Science and Technology. Submitted.

[4] Authors acknowledge the support by the STC Program of the National Science Foundation under Agreement No. ECS-9876771, the assistance of the Advanced Light Microscopy Core and the Electron Microscopy Core at the Wadsworth Center, which is supported in part by the NSF Grant DBI-0116551. This work was performed in part at the Cornell Nanofabrication Facility, which is supported by the NSF under Grant ECS-9731293.
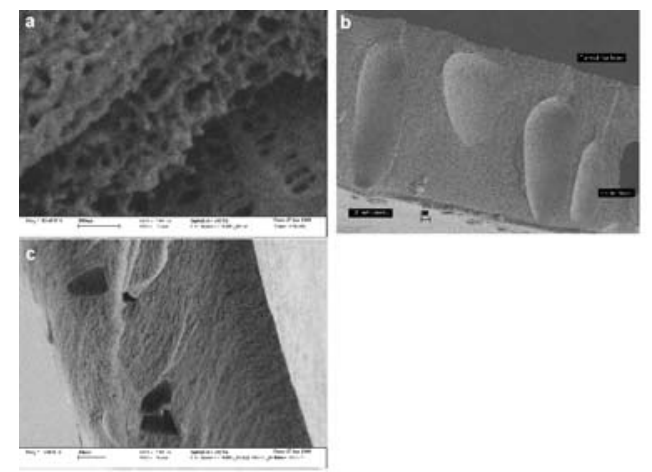

FIG.1. SEM of CA membranes showing the variation in their internal structures using different polymer solution concentrations: (a) 10\% CA in DMAc (space bar 300nm), (b) $25 \%$ CA in DMAc (space bar $3 \mu \mathrm{m}$ ), (c) $40 \%$ CA in DMAc (space bar $10 \mu \mathrm{m}$ ).
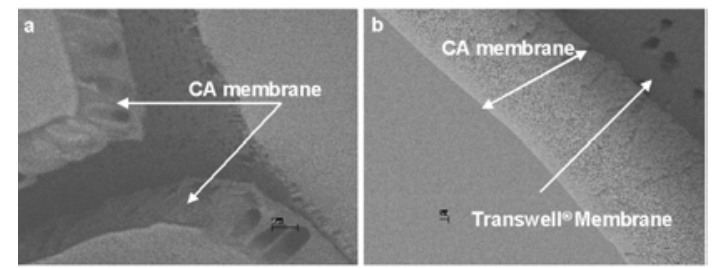

FIG. 2. SEM of fractured membranes showing the internal structure, both membranes were treated chemically using different conditions to promote (a) or prevent the formation of large voids (b).

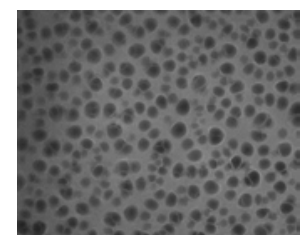

FIG. 3. Image of the stained membrane collected by confocal laser scanning microscopy showing fluorescence across the whole membrane except for the areas of the large voids. This is one in-focus $\mathrm{X}-\mathrm{Y}$ slice in the middle of the membrane out of the 25 slices collected over the Z-axis. The dark areas have no fluorescence and correspond to the voids. 